\title{
Dengue em criança: aspectos clínicos e epidemiológicos, Manaus, Estado do Amazonas, no período de 2006 e 2007
}

\author{
Dengue in children: clinical and epidemiological characteristics, \\ Manaus, State of Amazonas, 2006 and 2007
}

Lúcia Alves da Rocha ${ }^{1}$ e Pedro Luiz Tauil ${ }^{2}$

\begin{abstract}
RESUMO
O objetivo deste trabalho é apresentar os aspectos clínicos e epidemiológicos das crianças acometidas por dengue, em Manaus, AM, no período de 2006 a 2007. Foram analisados 482 casos de dengue confirmados laboratorialmente, em 2006, sendo 46,9\% nos menores de 15 anos, e 1.538 casos, em 2007, sendo 57,7\% nos menores de 15 anos. Os dados foram obtidos do Sistema Nacional de Agravos de Notificação (SINAN) de Manaus. As variáveis analisadas foram: idade, gênero, bairro de residência, mês do inicio dos sintomas, sorotipo e forma clínica da doença. Verificou-se um aumento estatisticamente significativo da proporção de casos em menores de 15 anos, bem como das formas graves entre 2006 e 2007 . Apesar de a forma hemorrágica ter sido quase três vezes mais freqüente em 2007, houve uma redução do coeficiente de letalidade. Os sorotipos identificados foram DEN-3 em 2006 e DEN-1, DEN-2 e DEN-3 em 2007. Em 2007, houve registro de casos em todos os meses do ano.
\end{abstract}

Palavras-chaves: Dengue. Crianças. Manaus. Amazonas.

\begin{abstract}
This study presents the clinical and epidemiological aspects of children affected with dengue, in Manaus, AM, in 2006 and 2007. There were 482 The aim of this study was to present the clinical and epidemiological characteristics of children affected by dengue, in Manaus, State of Amazonas, in 2006 and 2007. Analysis was conducted on 482 dengue cases with confirmation from laboratory tests, in 2006 ( $46.9 \%$ in children under 15 years of age), and 1,538 cases in 2007 ( $57.7 \%$ in children under 15 years of age). The data were obtained from the National System for Notifiable Diseases (Sistema Nacional de Agravos de Notificação; SINAN) in Manaus. The variables analyzed were: age, gender, district where subject lived, month when symptoms started, serotypes and clinical form of the disease. Statistically significant increases in the proportion of cases in children under 15 years of age and in the proportion of severe forms were found between 2006 and 2007. Although the hemorrhagic form was almost three times more frequent in 2007, the mortality rate was lower. The serotypes identified were DEN-3 in 2006 and DEN-1, DEN-2 and DEN-3 in 2007. In 2007, cases were recorded in every month of the year.
\end{abstract}

Key-words: Dengue. Children. Manaus. Amazonas.

Dengue continua sendo uma doença de elevada significância em Saúde Pública, tanto no Brasil como nos demais paises tropicais e subtropicais. Atualmente, considera-se que cerca de 2,5 a 3 milhões de pessoas vivem expostas ao risco de adquirir dengue ${ }^{9}$. É uma doença infecciosa aguda, cujo agente etiológico é um vírus pertencente à família Flaviridae, de genoma RNA, do qual são conhecidos quatro sorotipos (DEN-1, DEN-2, DEN-3, e DEN-4). É transmitido por picadas de insetos de hábitos urbanos, tendo como principal vetor, o Aedes aegyptí $i^{4}$. Apresenta um espectro clínico muito amplo, desde formas oligosssintomáticas até quadros graves com hemorragia e choque, podendo evoluir para o óbito.

\footnotetext{
1. Secretaria Municipal de Saúde, Manaus, AM. 2. Área de Medicina Social, Faculdade de Medicina, Universidade de Brasília, DF.

Endereço para correspondência: Dra. Lúcia Alves da Rocha. Av. Constantino Nery 2789, s706. Edifício Empire Center, Chapada. 69050-001 Manaus, AM.

Tel: 5592 3657-4615/3216-7751.

e-mail: lucia.rocha@pmm.am.gov.br

Recebido para publicação em 22/09/2008

Aceito em 13/01/2009
}

Em decorrência do processo de endemização do dengue no Brasil, após sua reemergência há 26 anos, vem ocorrendo uma mudança na sua distribuição etária, havendo um progressivo aumento da incidência em menores de 15 anos. Ao mesmo tempo, tem havido também um aumento da incidência das formas graves. Esta mudança do padrão epidemiológico da doença assemelha-se àquele observado no Sudeste Asiático ${ }^{3}$.

Em Manaus, capital do Estado do Amazonas, após a ultima epidemia com 18.595 casos, em 2001, o dengue adquiriu um caráter endêmico, com aumento da incidência nos períodos de chuvas, de janeiro a julho. Já foram identificados os sorotipos DEN-1, DEN-2 e DEN-3 e o grupo etário mais acometido tem sido o de 20 a 49 anos (Figura 1).

De 2000 a 2006, observa-se, na distribuição da média mensal do número de casos notificados, que a doença incidiu prioritariamente no primeiro semestre do ano (Figura 2).

0 presente trabalho tem como objetivo mostrar as características epidemiológicas e formas clínicas apresentadas pelas crianças, menores de 15 anos, acometidas por dengue autóctone, na Cidade de Manaus, AM, no período de 2006 a 2007. 


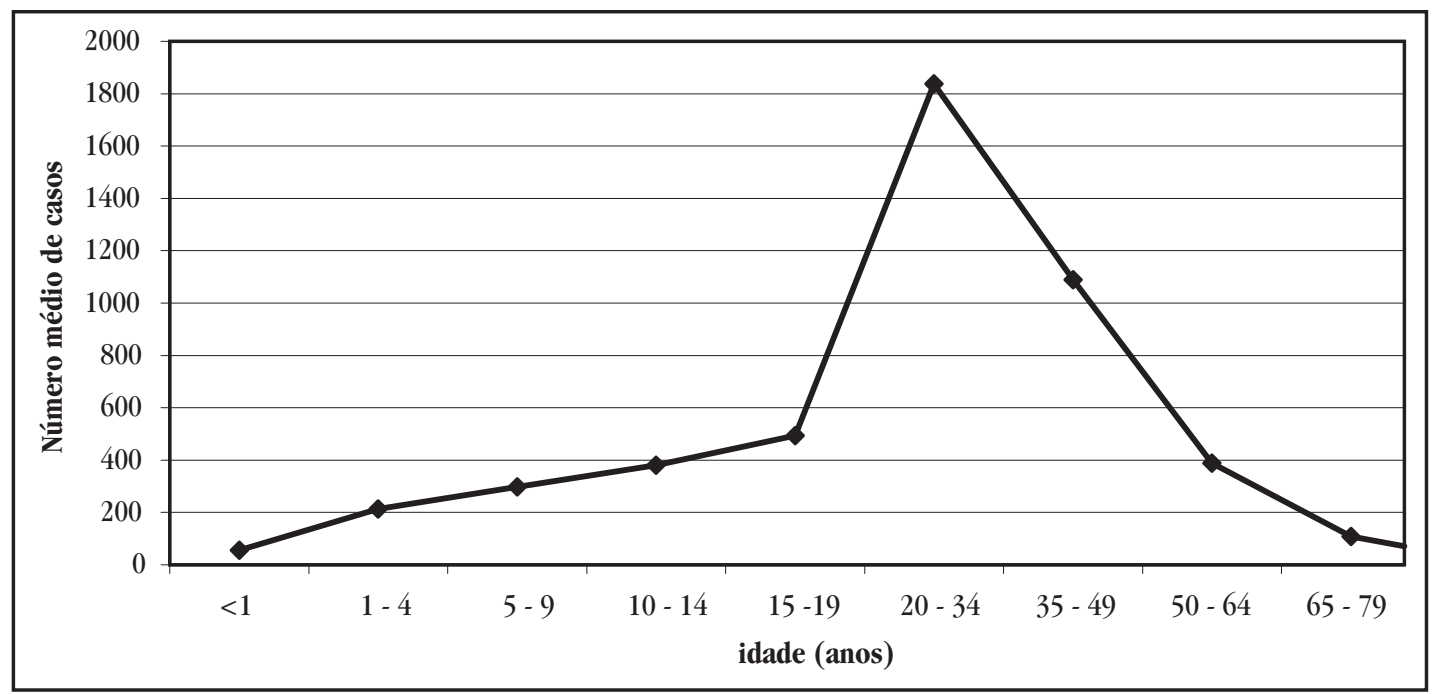

FIGURA 1

Distribuição do número médio de casos de dengue notificados por ano segundo o grupo etário, em Manaus, no período de 2000 a 2006. Fonte: SINAN/DEPAM/SEMSA

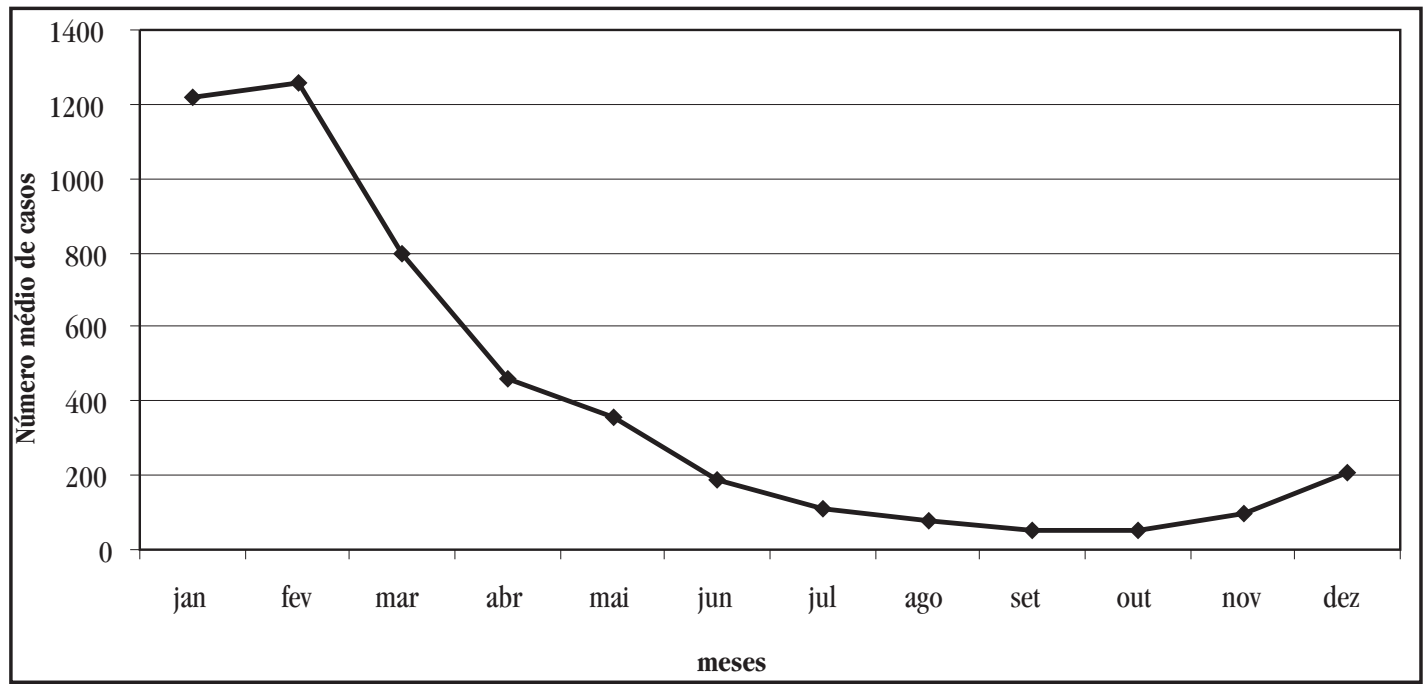

FIGURA 2

Distribuição da média mensal do número de casos de dengue notificados em Manaus, AM, no período de 2000 a 2006. Fonte: SINAN/DEPAM/SEMSA

\section{MATERIAL E MÉTODOS}

A pesquisa foi realizada em Manaus-AM, a partir dos dados disponíveis no Sistema de Informação de Agravos de Notificações (SINAN), sistema este que tem como objetivo coletar dados gerados rotineiramente do Sistema de Vigilância Epidemiológica/ Ministério da Saúde (SVS/MS), por meio das fichas de notificação das doenças de notificação compulsória, segundo a Portaria SVS/MS n ${ }^{0}$, de 21 de fevereiro de $2006^{7}$, de $1^{\circ}$ janeiro de 2006 a 31 de dezembro de 2007.

As variáveis analisadas foram: idade, gênero, bairro de residência, mês do inicio dos sintomas, sorotipo do vírus, quando disponível, e forma clínica da doença.

Entre os menores de 15 anos, foram avaliadas as categorias etárias consideradas pelo SINAN: menores de 1 ano, de 1 a 4 anos, de 5 a 9, e de 10 a 14 anos. Considerou-se, como o momento da infecção, a data do início dos sintomas. Esses dados permitiram o cálculo do coeficiente de incidência, por 100.000 habitantes, utilizando-se dados populacionais estimados pelo Instituto Brasileiro de Geografia e Estatística (IBGE) ${ }^{1}$.

A forma clínica foi obtida por meio da classificação final do caso: dengue clássico, febre hemorrágica do dengue (FHD), síndrome do choque do dengue e dengue com complicação. Os critérios considerados para a classificação clínica e laboratorial foram os da Organização Mundial de Saúde ${ }^{10}$ e do Ministério da Saúde ${ }^{11}$.

A confirmação laboratorial é realizada no Laboratório Central (LACEN) de Manaus, utilizando a técnica de ELISA para os exames sorológicos e o material para isolamento viral é encaminhado pelo LACEN ao laboratório de referência, o Instituto Evandro Chagas, em Belém do Pará.

Os dados obtidos foram organizados em figuras e tabelas, utilizando-se a planilha eletrônica Microsoft Office Excel 2003 
e o programa TabWin. 0 qui-quadrado foi o teste estatístico empregado para verificar a diferença nas proporções de incidência da doença, de formas graves e diferenças de gênero, nos grupos etários de $<15$ e de $\geq 15$ anos, entre 2006 e 2007. 0 uso dos dados disponíveis no SINAN/DEPAM/SEMSA foi autorizado pela Diretoria de Epidemiologia e Ambiente da Secretaria Municipal de Saúde, Manaus, AM.

\section{RESULTADOS}

Foram notificados no ano de 2006, 812 casos suspeitos de dengue, sendo que 445 (54\%) ocorreram em menores de 15 anos. Em 2007, esses valores foram de 3.622 casos suspeitos, sendo 2.080 (57,4\%) em menores de 15 anos. Em 2006 foram confirmados laboratorialmente, 482 casos, sendo 226 (46,9\%) em menores de 15 anos. Em 2007, foram confirmados 1.538 casos, sendo 888 (57,7\%) em menores de 15 anos. Esta diferença foi estatisticamente significativa $\left(\chi^{2}=17,03, \mathrm{p}<0,0001\right)$.
Na Figura 3, pode-se observar, entre os menores de 15 anos, o maior risco de adoecer por dengue entre os menores de um ano, fato este observado tanto em 2006 (114,1 por 100.000 habitantes) como em 2007 (210,7 por 100.000 habitantes). A Figura 4 mostra o aumento da incidência de febre hemorrágica do dengue/síndrome do choque do dengue nas diferentes faixas de menores de 15 anos, em 2006 e 2007. Enquanto em 2006 a maior incidência foi nos menores de 1 ano $(2,4$ por 100.000 habitantes), em 2007 houve, além de um aumento da incidência em todos os grupos etários estudados, um predomínio nas crianças de 10 a 14 anos.

A proporção de casos graves (incluindo dengue com complicação) em menores 15 anos passou de 4\% em 2006 para $11,6 \%$ em 2007. Esta diferença foi estatisticamente significativa $\left(\chi^{2}=10,7\right.$ e $\left.\mathrm{p}=0,001\right)$.

A proporção de incidência segundo o gênero, foi semelhante nos dois anos estudados, em torno de 50\% $\left(\chi^{2}=0,1 \mathrm{e} \mathrm{p}=0,8\right)$. A Figura 5 apresenta a distribuição mensal dos casos nos dois

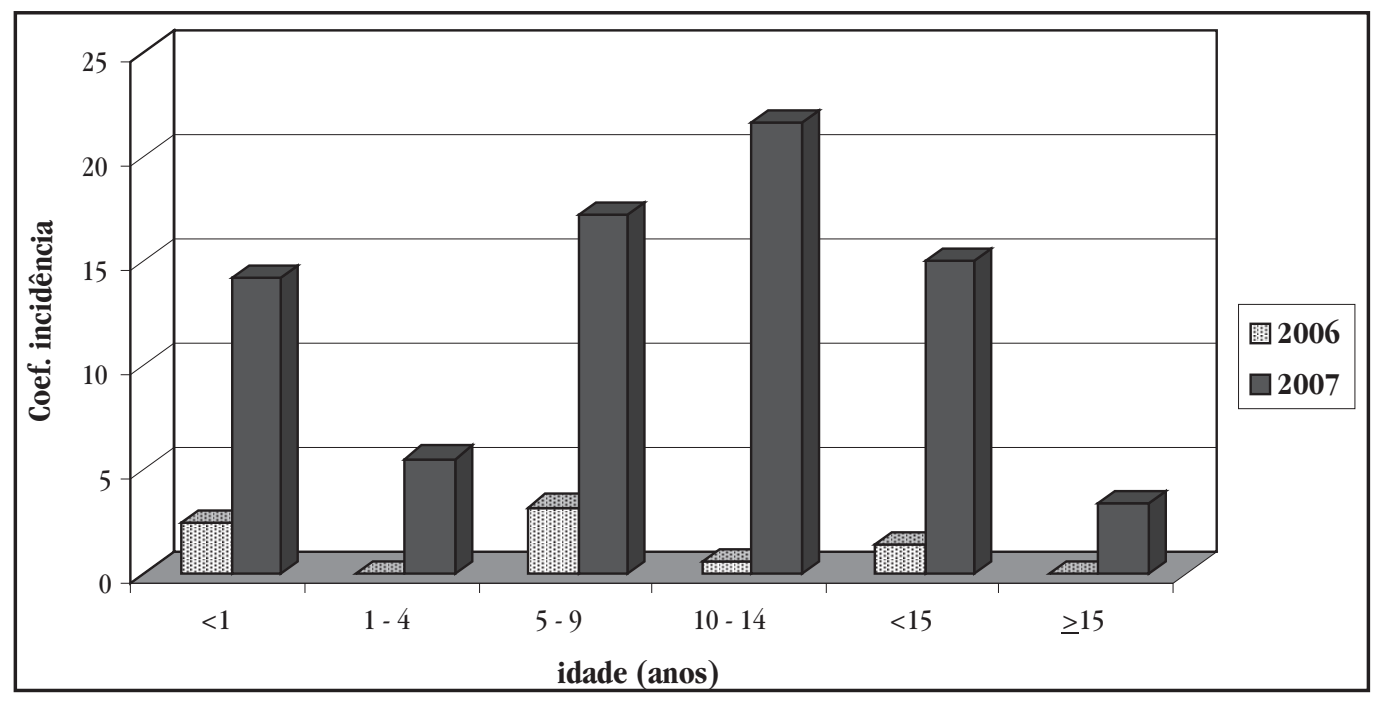

FIGURA 3

Coeficiente de incidência de dengue, por grupo etário (x 100.000 habitantes). Manaus, AM, 2006 e 2007. Fonte: SINAN/DEPAM/SEMSA.

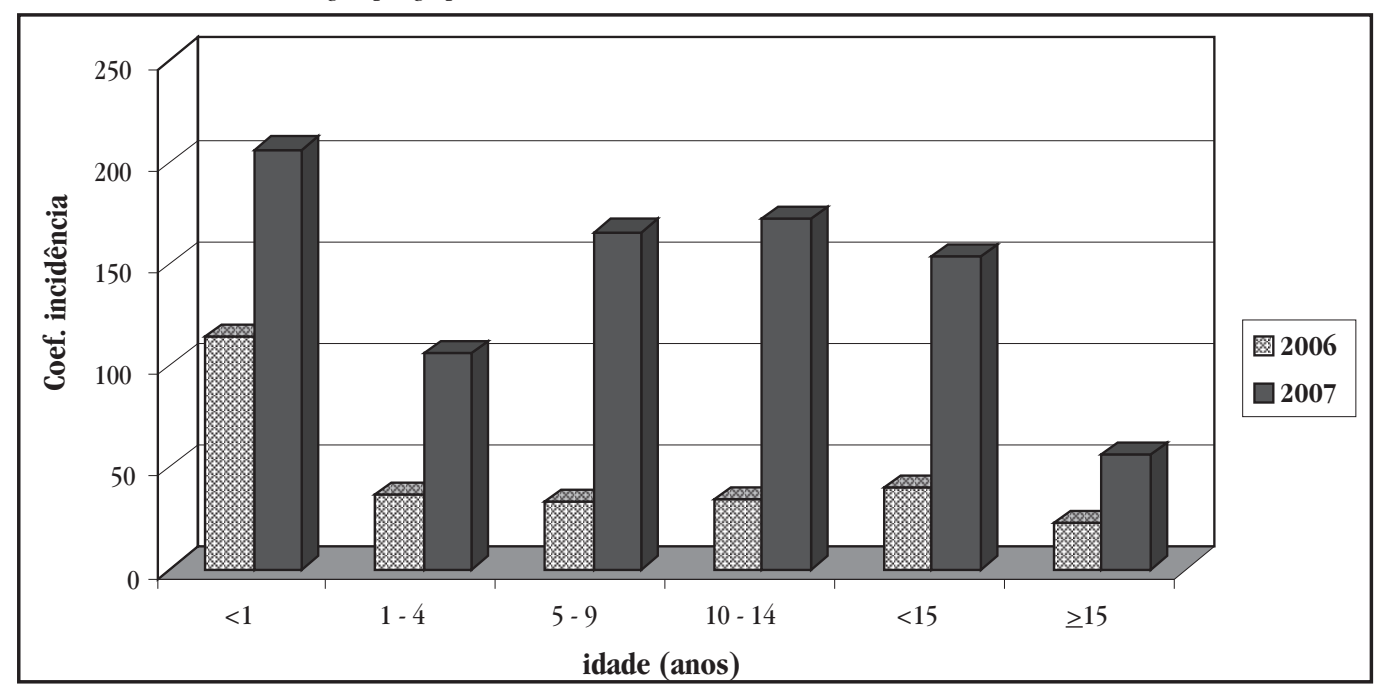

FIGURA 4

Coeficiente de incidência de febre hemorrágica do dengue/síndrome do choque do dengue, por grupo etário (x 100.000 habitantes). Manaus, AM, 2006 e 2007. Fonte: SINAN/DEPAM/SEMSA. 


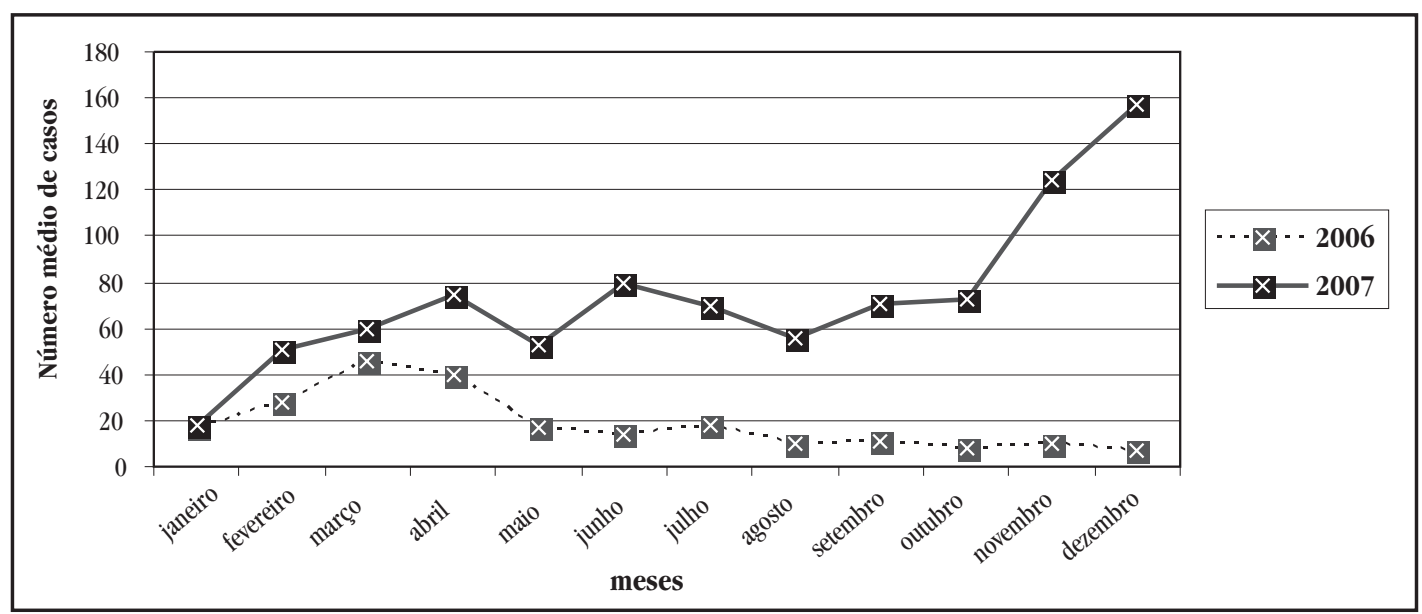

FIGURA 5

Distribuição mensal do número de casos confirmados de dengue, em menores de 15 anos. Manaus, AM, 2006 e 2007. Fonte: SINAN/DEPAM/SEMS

anos. Em janeiro de 2006 e 2007, não houve diferença quanto ao numero de casos, mas houve aumento da incidência em todos os outros meses do ano em 2007.

A distribuição percentual das formas clínicas encontra-se na Tabela 1. Verifica-se que houve um aumento de quase três vezes da participação das formas hemorrágicas (FHD e SCD) em 2007, em relação a 2006, passando de 3,5\% para 9,8\%. Quanto ao grau de gravidade da FHD, segundo os critérios da Organização Mundial de Saúde (OMS) ${ }^{4}$, em 2007, o grau II (49,4\%) foi o mais freqüiente (Figura 6) entre os 87 casos que se encontram com registro de gravidade no sistema, seguido do grau I (20,8\%), grau IV $(2,3 \%)$, grau III $(1,1 \%)$; houve 23 casos $(26,4 \%)$ sem registro do grau de gravidade e não houve caso registrado de síndrome do choque do dengue. Em 2006, apenas 6 casos de FHD e 2 com síndrome do choque do dengue (SCD) foram registrados no SINAN, sendo também o mais frequiente o grau II (50\%), seguido do grau I (12,5\%); nesse ano, houve 3 casos $(37,5 \%)$ sem registro do grau de gravidade.

De todos os casos confirmados por critério sorológico em 2006, apenas de 2 conseguiu-se fazer o isolamento viral, identificando o sorotipo 3; em 2007, foram identificados 3 casos do sorotipo 1, 5 do sorotipo 2 e 31 do sorotipo 3 (Tabela 2).

Com relação à letalidade, em menores de 15 anos, ocorreu 1 óbito (12,5\%), em 8 casos de FHD em 2006 e 4 óbitos (4,6\%) em 87 casos de FHD em 2007.

A distribuição espacial de dengue em menores de 15 anos, por bairro do município de Manaus, mostra que os bairros mais atingidos em 2006 foram Cidade Nova (11\%), Petrópolis (8,4\%), seguido pelo Japiim (6,2\%), Jorge Teixeira (6,2\%), Alvorada $(6,2 \%)$ e Compensa (4,9\%). Em 2007, os bairros mais afetados foram também, Cidade Nova $(16,9 \%)$, Japiim (7,4\%), Jorge Teixeira (7,1\%), Compensa (5,8\%) e Petrópolis (5\%).

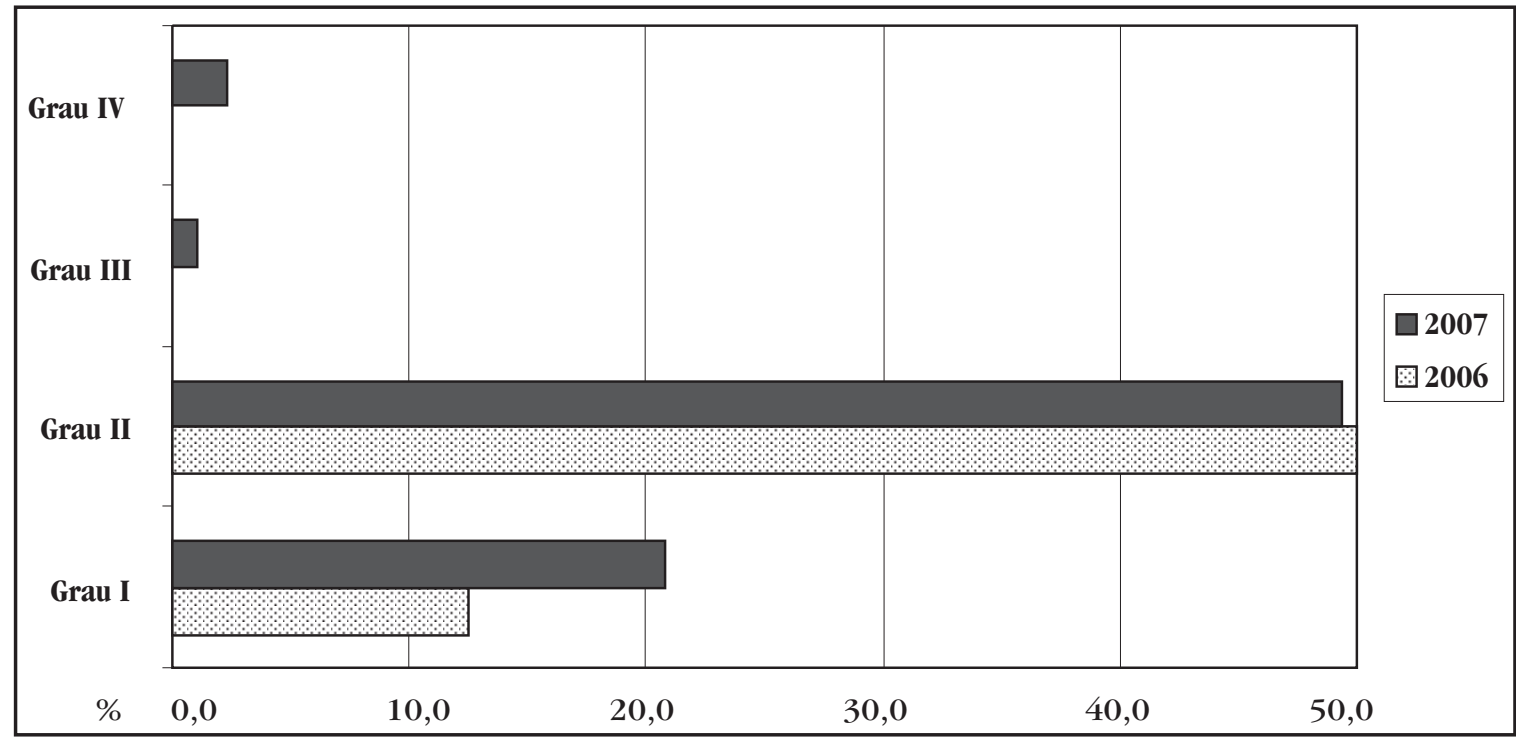

FIGURA 6

Distribuição percentual do grau de gravidade da febre hemorrágica do dengue, segundo critérios da Organização Mundial de Saúde, em menores de 15 anos. Manaus, AM, 2006 e 2007. Fonte: SINAN/DEPAM/SEMSA. 


\section{TABELA 1}

Frequiência relativa das formas clínicas de dengue nos dois grupos etários ( $<15$ e $\geq 15$ anos). Manaus, AM, 2006 e 2007 .

\begin{tabular}{lrrrrrr}
\hline & \multicolumn{2}{c}{2006} & & \multicolumn{2}{c}{2007} \\
\cline { 2 - 3 } \cline { 6 - 7 } Formas Clínicas & $<15$ & & & \multicolumn{2}{c}{$(\%)$} \\
\hline Dengue Clássico (DC) & 96,1 & 99,6 & & 88,4 & 91,5 \\
Febre Hemorrágica do Dengue & 2,6 & 0,0 & & 9,8 & 6,0 \\
Síndrome do Choque do dengue & 0,9 & 0,0 & & 0,0 & 0,0 \\
Dengue c/complicação & 0,4 & 0,4 & & 1,8 & 2,5 \\
\hline Total & 100,0 & 100,0 & 100,0 & 100,0 \\
\hline
\end{tabular}

TABELA 2

Frequiência dos sorotipos identificados pelo isolamento viral, nos dois grupos etários ( $<15$ e $\geq 15$ anos) com dengue. Manaus, AM, 2006 e 2007.

\begin{tabular}{lrrrrr}
\hline & \multicolumn{2}{c}{2006} & & \multicolumn{2}{c}{2007} \\
\cline { 2 - 3 } \cline { 5 - 6 } Sorotipos & $<15$ & $\geq 15$ & & $<15$ & $\geq 15$ \\
\hline Dengue 1 & 0 & 0 & & 3 & 1 \\
Dengue 2 & 0 & 0 & & 5 & 1 \\
Dengue 3 & 2 & 1 & & 31 & 6 \\
\hline Total & 2 & 1 & 39 & 8 \\
\hline
\end{tabular}

Fonte: SINAN/DEPAM/SEMSA.

\section{DISCUSSÃo}

0 registro da presença do Aedes aegypti em Manaus data de agosto de 1996, no bairro da Praça 14 de Janeiro (zona sul). Após isso, detectou-se o mosquito no bairro de São José (zona leste) e progressivamente alcançou toda a cidade. 0 primeiro caso de dengue autóctone foi registrado naúltima semana de março de 1998, quando então ocorreu a primeira epidemia de dengue na cidade, com 15.508 casos notificados e o sorotipo responsável foi o DEN-1 ${ }^{8}$.

A segunda epidemia ocorreu em 2001 com 18.595 casos notificados, estando presentes os sorotipos DEN-1 e DEN-2. A transmissão simultânea desses dois sorotipos facilitou 0 surgimento dos primeiros casos de febre hemorrágica do dengue em Manaus com o registro de $52 \operatorname{casos}^{8}$ A partir daí, o dengue vem se comportando de forma endêmica com aumento de incidência nos períodos de chuvas, que ocorre entre janeiro e julho, sendo os sorotipos circulantes o DEN-1, DEN-2 e DEN-3.

Porém, a análise dos resultados obtidos nos anos de 2006 a 2007, permitiu identificar uma mudança no comportamento da doença, tanto quanto à distribuição mensal, quanto à maior frequiência das formas graves e maior incidência em menores de 15 anos.

Os coeficientes calculados evidenciaram um aumento do risco de dengue nos menores de 15 anos com um coeficiente de incidência maior em menores de 1 ano (Figura 3), nos dois anos estudados. A frequiência da febre hemorrágica do dengue/síndrome do choque do dengue em 2007 foi elevada, principalmente no grupo de 5 a 14 anos (Figura 4). Observou-se uma semelhança com a distribuição etária dos casos de FHD ocorridos no Sudeste Asiático, onde estes também ocorrem principalmente em menores de 15 anos $^{3}$ e uma diferença em relação a Cuba e Porto Rico, onde os mais atingidos foram os adultos ${ }^{6}$. Ambos os gêneros em menores de 15 anos, foram igualmente afetados pelo dengue, assim como foi observado em epidemia na Índia ${ }^{2}$.
A Organização Mundial de Saúde considera aceitável uma letalidade por FHD de até $1 \%{ }^{10}$. 0 que se observou nesses dois anos de estudo é que a letalidade da FHD/SCD em Manaus, nos menores de 15 anos, está acima do aceitável, apesar de ter havido uma redução de 2006 (12,5\%) para 2007 (4,6\%).

Na distribuição espacial, a incidência de dengue em menores de 15 anos, em 2006, foi maior nos bairros da Cidade Nova, Petrópolis, Japiim e Jorge Teixeira. Em 2007, manteve-se nos mesmos bairros, atingindo ainda um percentual elevado no bairro da Compensa. Esses bairros localizam-se na zona norte, sul, leste e oeste respectivamente. Manaus, como um todo, possui todas as condições ecológicas para o desenvolvimento do mosquito vetor Aedes aegypti. Segundo a Organização Pan-americana de Saúde (OPAS), entre os fatores macrodeterminantes do dengue, destacam-se a densidade populacional moderada ou alta; padrões de assentamento inadequados; habitações com ausência de água encanada e com recipientes para armazenamento inadequadamente vedado; coleta de lixo deficiente, propiciando acúmulo de recipiente descartável e, finalmente, precária condições sócio-econômicas 5 . Esses bairros possuem pelo menos um desses fatores que, associado aos fatores climáticos da região amazônica, favorecem a proliferação do vetor. Portanto, é imprescindível manter continuamente as vigilâncias entomológica e epidemiológica com adoção de medidas de prevenção e controle da doença durante 0 ano todo.

Concluindo-se, verificou-se que entre 2006 e 2007, na Cidade de Manaus, AM, houve aumento significativo da proporção de casos de dengue em menores de 15 anos e da proporção de casos graves neste grupo etário. Não foi verificado diferença da proporção segundo o gênero.

\section{REFERÊNCIAS}

1. Instituto Brasileiro de Geografia e Estatística. Censos Demográficos e Contagem Populacional; para os anos intercensitários, estimativas preliminares dos totais populacionais, estratificadas por idade e sexo pelo MS/SE/Datasus. Período: 1998 - 2007, Ministério da Saúde, Brasília, 2007.

2. Kabilan L, Balasubramanian S, Keshava S M, Thenmozhi V, Sekar G, Tewari S C, Arunachalam N, Rajendran R, Satyanarayana K. Dengue disease spectrum among infants in the 2001 dengue epidemic in Chennai, Tamil Nadu, India. Journal of Clinical Microbiology 41: 3919-3921, 2003.

3. Nimmannitya $\mathrm{S}$. Dengue fever/dengue haemorrhagiclfever: case management. Tropical Medicine 36: 249-256, 1994.

4. Organização Mundial de Saúde. Dengue hemorrágica: diagnóstico, tratamento, prevenção e controle. Editora Santos, São Paulo, 2001.

5. Organização Panamericana da Saúde. Diretrizes relativas à prevenção e ao controle da dengue e da dengue hemorrágica nas Américas. Relatório da Reunião sobre Diretrizes para a Dengue, Washington, 1991.

6. Rigau-Peréz JG. Clinical manifestations of dengue hemorragic fever in Puerto Rico:1990-1991. Revista Panamericana de Saúde Pública 1:381-388, 1997.

7. Secretaria de Vigilância em Saúde. Guia de vigilância epidemiológica. Ministério da Saúde, 6a edição, Brasília, 2005.

8. Secretaria de Vigilância em Saúde. Portaria $n^{\circ}$ 5, de 21 de fevereiro de 2006. Diário Oficial da União, seção 1 - n 38, 22/02/2006, Brasília, 2006.

9. Sistema de Informação de Agravos de Notificações - SINAN/DEPAM/SEMSA. Secretaria Municipal de Saúde, Manaus, AM, 2007.

10. Torres EM. Dengue. Fiocruz, Rio de Janeiro, 2005.

11. World Health Organization. Regional Office for South-East Asia, New Delhi Comprhensive guidelines for prevention and control of dengue/DHF. WHO Regional Publication, SEARO n²9, 1999. 